\title{
Antiretroviral Therapy, CD4, Viral Load, and Disease Stage in Saudi HIV Patients: A Cross Sectional Study of Cases Diagnosed between 2001-2013
}

\author{
Adil Isaac ${ }^{1 *}$, Batol Ali $^{2}$, Sayed Qutb ${ }^{3}$ and Sana Flemban ${ }^{3}$ \\ ${ }^{1}$ King Saud Medical City-Riyadh, Saudi Arabia \\ ${ }^{2}$ King Saud Hospital-Jeddah, Saudi Arabia
}

${ }^{3}$ Saudi National AIDS Program, Ministry of Health, Saudi Arabia

\begin{abstract}
Objective: HIVIAIDS is increasing in the Kingdom of Saudi Arabia (KSA) and the Middle East. The proper use of antiretroviral therapy (ART), CD4 and VL tests helped control the HIV epidemic elsewhere. We aim in this study to analyze the use of ART, CD4 and VL and stage of presentation.
\end{abstract}

Design: A retrospective analytical descriptive cross sectional study.

Methods: Patients' medical records were used to obtain laboratory and other predefined variables that are not routinely reported in surveillance; namely: ART, CD4, VL, and CDC stage. Then we have described and analyzed these variables.

Results: A total of 997 cases from 8 HIVIAIDS care providers participated. Of whom 475 (47.6\%) on ART, and $522(52.4 \%)$ are not. Among those on ART: 247 (39.5\%) took Truvada combined with either efavirenz $147(14.7 \%)$ or Kaletra $100(10 \%)$. And $158(15.8 \%)$ are on Combivir with either efavirenz $32(3.2 \%)$ or Kaletra126 (12.6\%). And 70 $(7 \%)$ are on other combinations. The mean (Std deviation) baseline CD4 and Median (IQR) viral load are $401 \mathrm{cells} / \mu \mathrm{l}$ (322), and 4.6 log copies/ml (1.4) respectively.

At diagnosis $72 \%$ and $28 \%$ were asymptomatic and symptomatic respectively. Late presenters and those with AIDS were $50 \%$, and $20 \%$ respectively. Screening settings were low risk screening $35 \%$, high risk $44 \%$, and symptomatic $21 \%$.

Conclusions: ART use is in line with International Guidelines but those on ART are less than expected. Large proportions were LPs or have AIDS at diagnosis. Further data is needed to evaluate the medical care of patients with HIVIAIDS in the KSA.

Keywords: Saudi Arabia; HIV/AID; ART; CD4; CDC stage

\section{Introduction}

Since the advent of the HIV/AIDS epidemic, in the early 1980s, there was a response to early cases in the Kingdom of Saudi Arabia (KSA). The first such cases were reported in 1984, and since then surveillance of HIV commenced. However concerted activities related to HIV/AIDS were launched after the inception of the National AIDS Program (NAP) in 1996 to oversee surveillance and other HIV/AIDS public health activities [1].

However, monitoring of this program remains a challenge due to lack of dependable data collection systems. The likely reason is the diverse settings where HIV testing takes place coupled with lack of effective and seamless referral systems. However, since the last decade returns of prevalence and risk behavior data to the WHO UNAIDS were submitted regularly. However, it is widely believed that these reports underestimate the real burden of HIV/AIDS in the KSA [2].

The most recent UNAIDS country progress report published in 2014 revealed that incidence of HIV among Saudis has increased by $44 \%$ in 2013 compared to that of 2012 (550 vs. 400 new cases), which is consistent with the trends in many other MENA countries.

Recently published data revealed that while the overall prevalence of HIV/AIDS is consistently low, at less than $0.2 \%$, an epidemic is emerging among key populations at risk in KSA and the MENA region. The epidemic is heterogeneous within and across countries in the region. Also, the overall incidence and prevalence of HIV/AIDS is widely thought to be under stating the real burden of disease due to incomplete surveys and the widespread stigmatization in this region [3-5]. In addition, clinic based cohort studies suggested that patients are presenting late with AIDS. It is hoped that with better surveys, and programmatic work to reduce the stigma associated with HIV/AIDS, the epidemic in this region would be better understood [6,7].

There is scarce published data on the use of antiretroviral therapy, CD4, VL, or emergence of resistance to ART in the KSA. Presently, there is no agreed National Treatment Guidelines but International Guidelines are being used on ad hoc basis. Hence clinical practice is likely to be diverse for the lack of agreed drug treatment standards [7]. Int he World Development Report 2013, the KSA is considered a high-income country. ART is available free of charge but options for treatment-experienced patients are still limited. Also, resistance tests are not available in most treating centers, nor is co receptor and HLA B5701 screening (personal communication).

*Corresponding author: Adil Isaac, King Saud Medical City-Riyadh, PO BOX 366428, Riyadh11393, KSA, Saudi Arabia, Tel: 0557 919695; E-mail: Adilisaac@yahoo.com

Received March 09, 2015; Accepted March 25, 2015; Published April 10, 2015

Citation: Isaac A, Ali B, Qutb S, Flemban S (2015) Antiretroviral Therapy, CD4 Viral Load, and Disease Stage in Saudi HIV Patients: A Cross Sectional Study of Cases Diagnosed between 2001-2013. J AIDS Clin Res 6: 447. doi:10.4172/2155 6113.1000447

Copyright: () 2015 Isaac A, et al. This is an open-access article distributed under the terms of the Creative Commons Attribution License, which permits unrestricted use, distribution, and reproduction in any medium, provided the original author and source are credited. 
Therefore, studying baseline CD4 count and ART is important to evaluate the medical care of people living with HIV/AIDS, especially in the light of reports that mortality associated with HIV/AIDS is high in KSA and elsewhere in the MENA region. In the era of ART late presentation and poor access to services, among other factors, are associated with high mortality [8-10].

In this study, and for the first time from the KSA, we described the pattern of ART use, baseline CD4 and viral load, along with the disease stage at diagnosis. We tried to answer the following questions:

-What ART the the patients are and weather it is inline with International Treatment Guidelines?

- What is the baseline CD4 and VL? And whether late presentation is an issue?

We anticipated our study would serve as a baseline to measure future progress and a first step to fill in the many gaps in the information about medical care of people living of HIV/AIDS in (KSA). Also, we hope to feedback providers to standardize care in order to reduce morbidity and mortality in people living with HIV/AIDS [11-13].

\section{Methods}

We collected individual data on new HIV patients reported in the KSA in 2001-2013 to the HIV surveillance system. The NAP Immunity Unit in Riyadh coordinated the surveillance and obtained IRB approval.

Data reported by clinicians to the HIV regional coordinators was linked to obtain further variables that are not reported routinely in the annual survey: ART used CD4, VL and CDC stage at diagnosis. Regional HIV coordinators were trained to collect the said data.

Data was available mostly from the Western, Eastern and Southern regions, and limited data was obtained from the Northern and Central region. However, the majority of patients living with HIV in KSA reside in the participating regions.

\section{The following variables were collected}

Age, gender, nationality, marital status, region of residence, CDC stage, mode of transmission, CD4, Viral load, use of ART. Also, dates of notification, first HIV clinic visit, and start of ART.

The data was stored in an excel sheet, and then transferred to IBM SPSS-21 statistics package for analysis. In this article we mainly describe and analyze the ART, CD4, VL, and disease stage variables

\section{Results}

Data from a total of 977 patients living with HIV was available from 8 providers in all regions of KSA. Their respective regions of residence are as follows: Western 52\%, Southern 23\%, and Eastern 24\% and other regions $1 \%$.

The number of patients on ART is 475 (47.6\%), and those who are not on therapy are $522(52.4 \%)$. Table 1 shows the frequencies of ART used. Efavirenz/Truvada is the most frequently used combination (14.7\%) followed by Lopinavir/Ritonavir (Kaletra)/Combivir (12.6\%). Those taking efavirenz/Combivir and Kaletra/Truvada were 3.2\%, and $10 \%$ respectively.

The mean nadir CD4 and baseline VL were $401 \mathrm{cell} / \mu \mathrm{l}$ (std deviation 322) and $4.6 \mathrm{log}$ copies/ml (IQR 1.4). The proportions of low ( $<200$ cells/cu mm) CD4, and high VL ( $>5 \mathrm{log}$ ) were $28 \%$ and $42 \%$ respectively (Table 2 ).

\begin{tabular}{|l|c|c|}
\hline & Percent & Cumulative Percent \\
\hline Efavirenz/Truvada & 14.7 & 14.7 \\
\hline Kaletra/Truvada & 10.0 & 24.8 \\
\hline Efavirenz/Combivir & 3.2 & 28.0 \\
\hline Kaletra/Combivir & 12.6 & 40.6 \\
\hline Others & 7.0 & 47.6 \\
\hline Not_on_ART & 52.4 & 100.0 \\
\hline Total & 100.0 & \\
\hline
\end{tabular}

Table 1: Frequencies of antiretroviral therapy (ART) use.

\begin{tabular}{|c|c|c|c|c|}
\hline & \multicolumn{2}{|c|}{ CD4Cat } & \multirow{2}{*}{ Total } \\
\hline & & $>200$ & $<200$ & \\
\hline \multirow{2}{*}{ Viral Load Category } & $<100000$ & $67.5 \%$ & $32.5 \%$ & $57.7 \%$ \\
\hline & $>100000$ & $55.1 \%$ & $44.9 \%$ & $42.3 \%$ \\
\hline \multicolumn{2}{|l|}{ Total } & $71.7 \%$ & $28.3 \%$ & $100 \%$ \\
\hline
\end{tabular}

Table 2: Cross tabulation of CD4 and viral load categories.

\begin{tabular}{|l|c|c|c|c|}
\hline \multicolumn{2}{|c|}{} & \multicolumn{3}{|c|}{ HIV Test Setting } \\
\cline { 3 - 5 } & & Screening & High risk & Symptomatic \\
\cline { 3 - 5 } & & $\%$ & $\%$ & $\%$ \\
\hline \multirow{3}{*}{ CDC stage at diagnosis } & A & $29.9 \%$ & $36.1 \%$ & $4.8 \%$ \\
\cline { 2 - 5 } & B & $2.1 \%$ & $4.8 \%$ & $6.5 \%$ \\
\cline { 2 - 5 } & C & $1.8 \%$ & $3.3 \%$ & $9.8 \%$ \\
\hline
\end{tabular}

Pearson Chi Square $\mathrm{P}<0.0001$

Table 3: HIV test settings and CDC disease stages.

Table 3 displays the CDC stage and test setting of the participants at the time of diagnosis. The largest proportions are CDC stages A; asymptomatic patients in the test setting of high-risk category, which included prisoners, and PWID and screening of contacts of known cases.

However, the late presenters $(C D 4<350)$ and those with advanced HIV disease (CD4<200 or AIDS regardless of the CD4 count) were $50 \%$ and $20 \%$ respectively. In Figure 1 late presenters, by CD4 criteria, are the dominant group in both the Western and Southern regions unlike

Nadir CD4 Categories by Regions of Residence

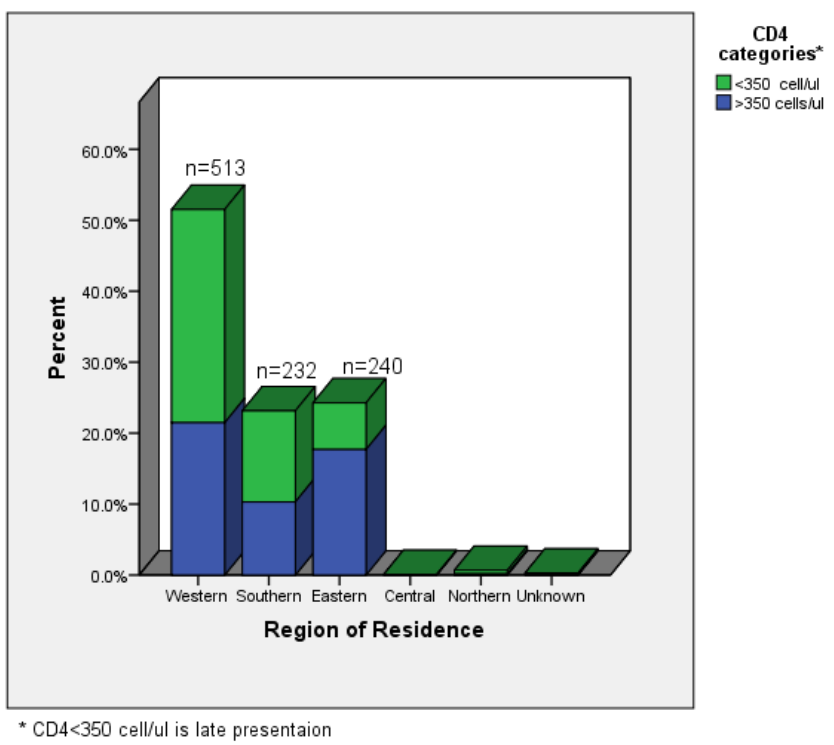

Figure 1: Nadir CD4 Categories by Regions of Residence. 


\section{Reported Mode of Transmisison by Region of Residence}

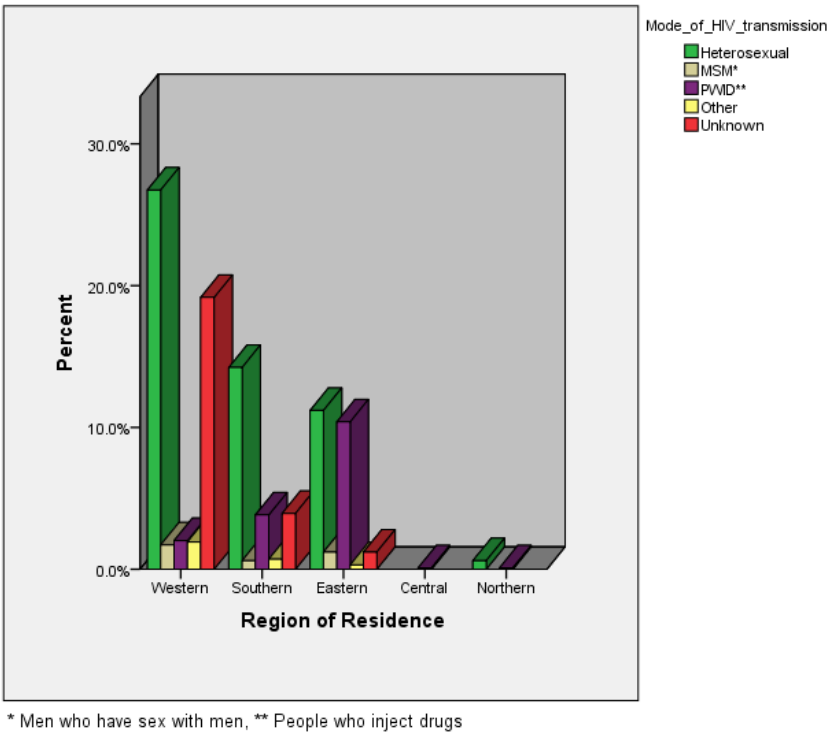

Figure 2: Reported Mode of Transmission by Region of Residence.

the case in the Eastern region.

The risk categories by regions revealed that the Western region followed by the Easter and Southern have the highest proportion of people living with HIV/AIDS; at 54\%, 24\% and 23\% respectively.

In addition, our results showed that the commonest reported mode of transmission is heterosexual, followed by those whose risk of transmission is reported as unknown except in the Eastern region where PWID comes second (Figure 2).

\section{Discussion}

Our results suggested that $47.6 \%$ of the patients are taking ART and the greater majority is taking drugs that are recommended, as first line or alternatives, by international authoritative guidelines. And a small group is on unusual ART combinations. We have also shown that mean CD4 and median viral load at diagnosis are $401 \mathrm{cell} / \mu \mathrm{l}$ (std deviation 322), and $4.6 \log$ (IQR 1.4). However, those who presented late $(C D 4<350)$ and those with advanced HIV disease $(C D 4<200$ or AIDS regardless of the CD4 count), were $50 \%$ and $20 \%$ respectively.

The high proportion of late presenters should prompt the HIV screening program to identify their characteristics and target them with testing. This is because late presentation is known to compromise the health of patients living with HIV. Therefore, a new study to further evaluate their characteristics is needed.

In addition, and consistent with previously published reports, most of the patients live in the Western Region of the KSA, and they were asymptomatic at diagnosis [14-17]. The main city in the latter region is Jeddah which is a see port and had always had the highest prevalence of people living with HIV/AIDS in KSA.

To our knowledge this is the first time that the use of ART has been described in HIV/AIDS patients in the KSA and there is no similar data in KSA or the MENA region to compare our results with. In our sample $80 \%$ of patients were diagnosed between 2006-2013 (data not shown), and the study was conducted in 2013, a period in which all authoritative International HIV Treatment Guidelines at least recommended the use of ART below a CD4 threshold of $\leq 350$ and more liberally since 2011 . With $47.6 \%$ on ART in our late-presenter dominated sample, we believe this is less than expected if International Guidelines are followed in the KSA. However, in this analysis we cannot confirm or rule out that ART was offered and declined or that ART offering has been increasing over the wide period of diagnose 2001-2013 [15,18].

The drug combinations used in ART reflected the most updated international treatment guidelines with the exception of the first line protease inhibitors (PIs), which is no longer Lopinavir/Ritonavir (Kaletra) as the case in our results. Rather, all International Guidelines recommend Darunavir/Ritonavir or Atazanavir/Ritonavir as first line PIs. Furthermore, no patient was taking an integrase inhibitor such as Raltegravir, and the nucleoside backbones did not include Abacavir singularly or co-formulated with Lamivudine, most likely due to the lack of availability of both the drugs and HLA B5701 typing [14]. In addition, the Co receptors Inhibitor class is not reported in our data; again most likely due to the unavailability of co-receptor test (personal communication). These tests and resistance tests are a prerequisite to provide modern high standard treatment to patients living with HIV. Also, by making available drug options for ART- experienced patients which is very important due to the likeliness, if not the certainly, that patients have accumulated resistance to ART. The latter has been available in the KSA since the mono-therapy era. Hence only different ART classes and HIV resistance tests can help salvage ART-experienced patients [13].

The means/median CD4 and VL counts reflected the mixed patient population ranging from routine premarital screening to sick patients in hospitals. This is consistent with a single previous study [7]. However, those who presented late on CD4 or clinical criteria amounted to $70 \%$. These results, especially if they were to be reproduced in other studies, will have an implication on the HIV screening program in the KSA. There will be a need for further targeting of the high-risk population through identifying the characteristics of the late presenters $[11,12,19]$.

The Central region mainly the city of Riyadh is underrepresented in our sample as data could only be obtained from providers under the Ministry of Health, and non-Ministry of health providers dominates HIV/AIDS care in Riyadh. Therefore, we advise caution in generalizing our result in this region. Also our sample had 5-15\% missing values, and we replaced them when $>5 \%$ by multiple Imputations.

In line with previous surveillance reports, the commonest reported mode of transmission is heterosexual followed by unknown mode. This most likely reflects the dire social and legal consequences of admitting MSM and PWID statuses, and hence it is underreported.

In conclusion, our results showed that while ART drugs in use are generally in line with International Guidelines, the proportion taking them is probably less than anticipated. And those who presented late are considerably higher than desired. Therefore, we recommend that ART and HIV testing should be offered more actively. Further and better surveillance data that include new variables such as CD4 is needed to monitor drug treatment of HIV/AIDS. This will expand on our findings, and provide clearer insight into the drug therapy of HIV/ AIDS in the KSA.

\section{References}

1. Al-Mazrou YY, Abouzeid MS, Al-Jeffri MH (2005) Impact of health education on knowledge and attitudes of Saudi paramedical students toward HIVIAIDS. Saudi Med J 26: 1788-1795. 
Citation: Isaac A, Ali B, Qutb S, Flemban S (2015) Antiretroviral Therapy, CD4, Viral Load, and Disease Stage in Saudi HIV Patients: A Cross Sectional Study of Cases Diagnosed between 2001-2013. J AIDS Clin Res 6: 447. doi:10.4172/2155-6113.1000447

Page 4 of 4

2. Mazroa MA, Kabbash IA, Felemban SM, Stephens GM, Al-Hakeem RF, et al (2012) HIV case notification rates in the Kingdom of Saudi Arabia over the past decade (2000-2009). PLoS One 7: e45919.

3. Al-Ghanim SA (2005) Exploring public knowledge and attitudes towards HIV/ AIDS in Saudi Arabia. A survey of primary health care users. Saudi Med J 26 812-818.

4. Al-Mazrou YY, Abouzeid MS, Al-Jeffri MH (2005) Knowledge and attitudes of paramedical students in Saudi Arabia toward HIVIAIDS. Saudi med j 26: 11831189.

5. Badahdah A (2005) Saudi attitudes towards people living with HIVIAIDS. Int J STD AIDS 16: 837-838.

6. Mumtaz GR, Riedner G, Abu-Raddad LJ (2014) The emerging face of the HIV epidemic in the Middle East and North Africa. Curr Opin HIV AIDS 9: 183-191.

7. Alrajhi AA, Halim MA, Al-Abdely HM (2006) Presentation and reasons for HIV-1 testing in Saudi Arabia. Int J STD AIDS 17: 806-809.

8. Madani TA, Al-Mazrou YY, Al-Jeffri MH, Al Huzaim NS (2004) Epidemiology of the human immunodeficiency virus in Saudi Arabia; 18-year surveillance results and prevention from an Islamic perspective. BMC Infect Dis 4: 25.

9. Mokdad AH, Jaber S, Aziz Ml, AlBuhairan F, AlGhaithi A, et al. (2014) The state of health in the Arab world, 1990-2010: an analysis of the burden of diseases, injuries, and risk factors. Lancet 383: 309-320.

10. Badie BM, Nabaei G, Rasoolinejad M, Mirzazadeh A, McFarland W (2013) Early loss to follow-up and mortality of HIV-infected patients diagnosed after the era of antiretroviral treatment scale up: a call for re-invigorating the response in Iran. Int J STD AIDS 24: 926-930.
11. When To Start Consortium, Sterne JA, May M, Costagliola D, de Wolf $F$, et al. (2009) Timing of initiation of antiretroviral therapy in AIDS-free HIV-1-infected patients: a collaborative analysis of 18 HIV cohort studies. Lancet 373: 1352 1363

12. Lawn SD, Harries AD, Wood R (2010) Strategies to reduce early morbidity and mortality in adults receiving antiretroviral therapy in resource-limited settings. Curr Opin HIV AIDS 5: 18-26.

13. Jamjoom GA, Azhar El, Madani TA, Hindawi SI, Bakhsh HA, et al. (2010) Genotype and antiretroviral drug resistance of human immunodeficiency virus-1 in Saudi Arabia. Saudi Med J 31: 987-992.

14. Labarga $P$ (2012) New DHHS guidelines recommend antiretroviral therapy to all HIV-infected persons. AIDS Rev 14: 154.

15. Labarga $P$, Blanco $F(2012)$ New antiretroviral treatment guidelines from the IAS-USA panel. AIDS Rev 14: 290.

16. Williams I, Churchill D, Anderson J, Boffito M, Bower M, et al. (2012) British HIV Association guidelines for the treatment of HIV-1-positive adults with antiretroviral therapy 2012. HIV med 13 Suppl 2: 1-85.

17. Doherty M, Ford N, Vitoria M, Weiler G, Hirnschall G (2013) The 2013 WHO guidelines for antiretroviral therapy: evidence-based recommendations to face new epidemic realities. Curr Opin HIV AIDS 8: 528-534.

18. Gazzard B, Bernard AJ, Boffito M, Churchill D, Edwards S, et al. (2006) British HIV Association (BHIVA) guidelines for the treatment of HIV-infected adults with antiretroviral therapy (2006). HIV Med 7: 487-503.

19. Kober C, Johnson M, Fisher M, Hill T, Anderson J, et al. (2012) Non-uptake of highly active antiretroviral therapy among patients with a CD4 count $<350$ cells/ $\mu$ in the UK. HIV Med 13: 73-78. 